\section{IgG4 Syndrome Presenting as Leptomeningitis in a Young Woman}

\section{To the Editor:}

Immunoglobulin G4-related disease (IgG4-RD) is a recognized systemic condition that can affect almost every organ system ${ }^{1}$. The most commonly involved organs are the pancreas, salivary glands, and biliary tree. The common presentation with central nervous system (CNS) involvement is hypophysitis and pachymeningitis, but rarely leptomeningitis ${ }^{2}$. Only 3 reported cases of leptomeningitis have been reported, all of them presenting as cognitive decline, and 2 associated with rheumatoid arthritis (RA) ${ }^{3}$. We describe a patient with unilateral hearing loss and a right frontal mass on magnetic resonance imaging (MRI) who was subsequently diagnosed with IgG4-related leptomeningitis after biopsy. This is a unique case of IgG4-related leptomeningitis because of its presentation and lack of association with RA or positive serological markers.

A 43-year-old woman with thyroid follicular cancer, post-thyroidectomy, had progressive right-side hearing loss for 2 months. She was also noted to have gait difficulty, pins-and-needles sensations in the feet, and facial droop on the left side. Physical examination showed that she was alert and oriented to time, place, and person. Her cranial nerves were intact except for the left-sided facial droop, 3+ biceps, triceps, and knee reflexes on the left side, and 4/5 strength on the left upper and lower extremity. Romberg sign was positive.

Contrast-enhanced MRI of the brain showed 2 enhancing dural-based lesions (Figure 1). One was in the high convexity of the right frontal region and a second at the skull base, which extended along the tentorium cerebelli bilaterally and inferiorly along the bilateral petroclival ligament and into the bilateral internal auditory canals. This explained her hearing loss. There was also extension along the dural reflections of the posterior fossa to the level of the foramen magnum and into the cervical spinal canal. The right frontal mass was removed by the neurosurgeon because it was thought to be a benign meningioma. However, pathological study revealed a lymphoplasmacytic infiltrate in the leptomeninges (Supplementary Figures 1 and 2, available online at jrheum.org). Special stains for acid-fast bacilli, fungi, and spirochetes were negative. More than 20 (27-48) IgG4-reactive plasma cells per high-power field were found in multiple foci of dense inflammatory infiltrates. The ratio of IgG4+/IgG cells was more than $40 \%$ (Figure 2). An extensive rheumatological examination included tests for antinuclear antibodies, SS-A, SS-B, dsDNA antibodies, rheumatoid factor, complement levels, erythrocyte sedimentation rate, C-reactive protein, and anticyclic citrullinated peptide, which were all negative. The patient also had IgG subtypes (1-4) measured in her plasma and all were found to be normal. Lumbar puncture was done with no evidence of IgG4 oligoclonal bands.

After surgery, the patient was treated first with intravenous dexamethasone and then oral dexamethasone. Two months later, the patient showed improvement in her balance, although her hearing did not return to baseline. Moreover, the diffuse dural thickening in the skull base seen on the MRI brain at presentation was not evident on the repeat computed tomography of the head.

The CNS manifestations of IgG4-RD usually present as pachymeningitis $^{2}$, but there are many other CNS presentations that can occur ${ }^{4,5}$. Mehta, et al reported the first case of IgG4-related leptomeningitis ${ }^{6}$ and there were clear differences in our case from the one reported by Mehta. The initial symptom was unilateral hearing loss instead of rapid cognitive decline. The MRI in our case showed dural-based lesions rather than diffuse leptomeningeal enhancement, mimicking MRI images of pachymeningitis. However, the pathology revealed a lymphoplasmacytic infiltrate limited to leptomeninges, without dural involvement.

Our patient also had an abnormal gait similar to the previously reported case. Such neurological deficits reflect mechanical compression of nerve structures, leading to neurological deficits ${ }^{7}$. The most common presenting neurological symptoms in patients with IgG4-RD are headache, cranial nerve palsies, vision problems, motor weakness, limb numbness, sensorineural hearing loss, and seizures. The definitive diagnosis of IgG4-RD requires biopsy demonstrating characteristic pathology along with IgG4 immunostaining.

It has been demonstrated that meningeal involvement in IgG4-RD results in disruption of the blood-cerebrospinal fluid (CSF) barrier (which likely depends on the meningeal inflammatory infiltrates), and high concentrations of oligoclonal IgG bands and IgG4 are found in the CSF. Della- Torre, et al have demonstrated that IgG4 hypertrophic pachymeningitis (IHP) can be

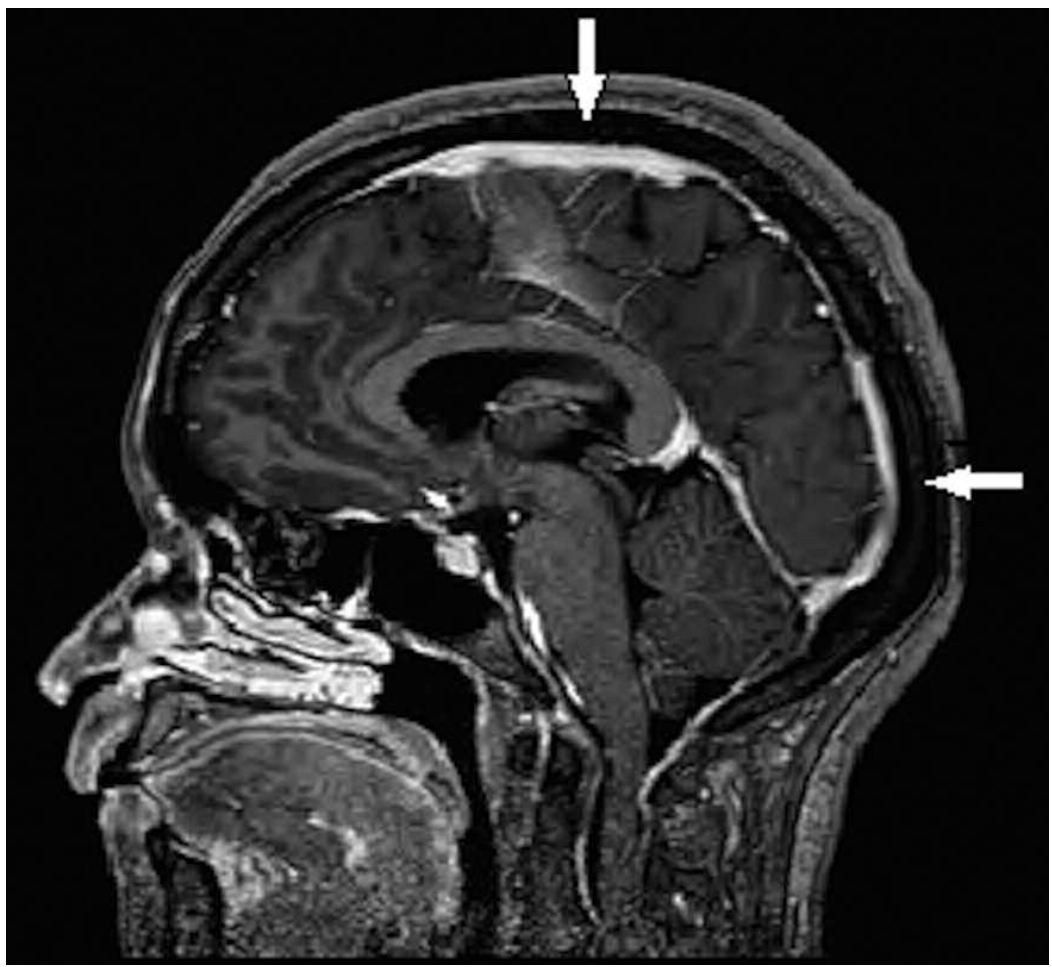

Figure 1. Contrast-enhanced magnetic resonance imaging (MRI) of the brain showed 2 enhancing dural-based lesions: one in the high convexity right frontal region and a second at the skull base (arrows). 


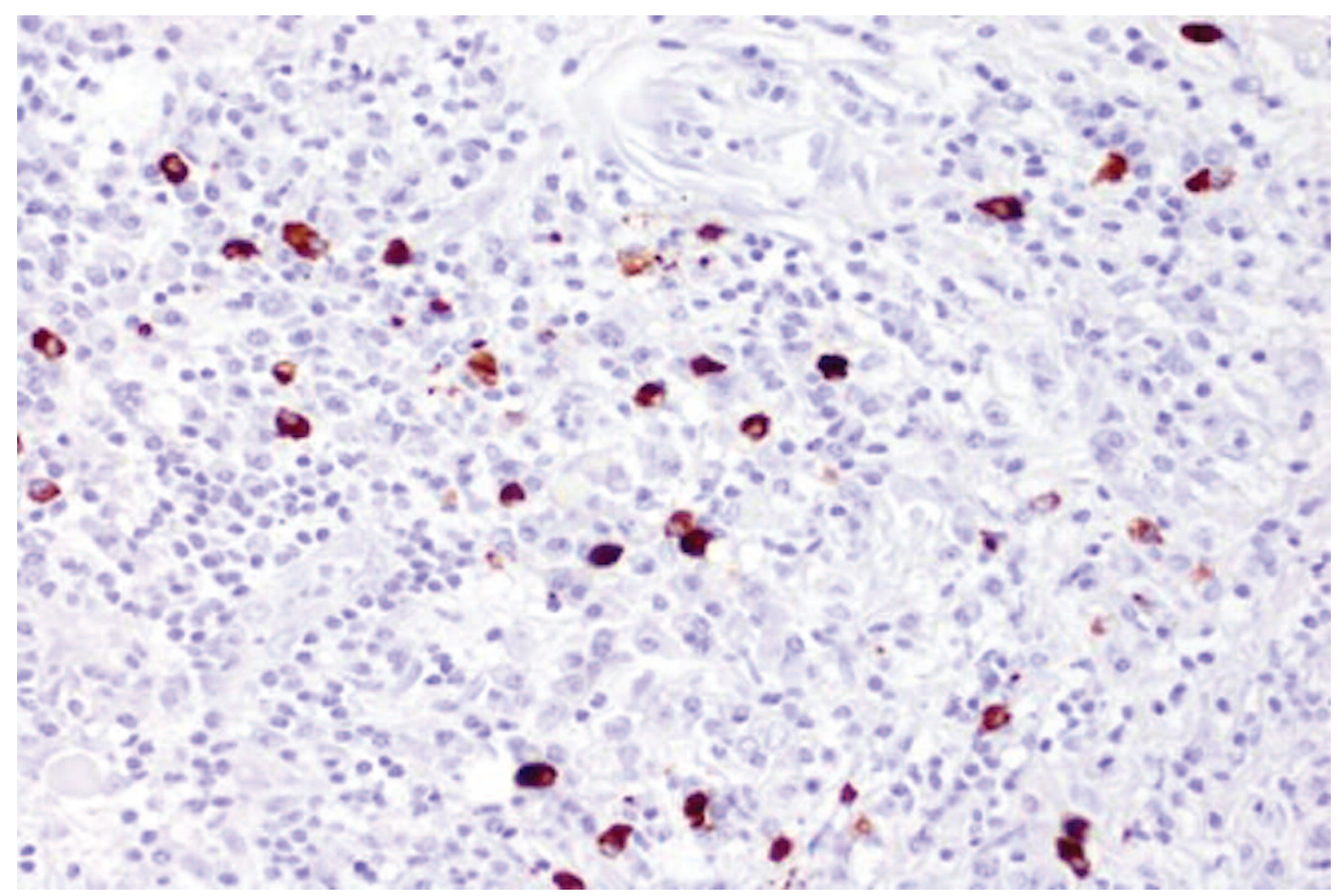

Figure 2. Lymphoplasmacytic infiltrate in meningeal tissue with organizing fibrosis. There are 27 to 48 IgG4-reactive plasma cells per high power field (multiple fields counted). Acid-fast and Congo red stain were negative; $\operatorname{IgG} 4+\operatorname{IgG}$ ratio $>40 \%$. $\operatorname{IgG} 4$ : immunoglobulin G4.

diagnosed with CSF IgG4 and $\mathrm{IgG} 4$ indices measurement ${ }^{\gamma}$ if biopsy is contraindicated, technically risky, or uninformative. All the patients with IHP had elevated serum levels of IgG4. Our patient had normal CSF analysis, normal serum IgG4 level, and involvement of leptomeninges as opposed to pachymeninges. Diagnosis in our patient was based mainly on histopathological analysis. Further studies have to be done in patients with leptomeningitis and normal serum IgG4 levels to use CSF indices for diagnostic and prognostic purposes.

It is important to consider IgG4-RD leptomeningitis in any patient presenting with an unusual neurological manifestation and characteristic findings on MRI. Establishing the diagnosis of IgG4-RD is important to initiate immunosuppressive therapy early to prevent further neurologic sequelae.

SOWMINI MEDAVARAM, MD, Internal Medicine Resident, Newark Beth Israel Medical Center; FEIFEI XUE, MD, Internal Medicine Resident,

Newark Beth Israel Medical Center; ROBERT LAHITA, MD, Chairman of Medicine, Newark Beth Israel Medical Center, Newark,

New Jersey, USA. Address correspondence to Dr. S. Medavaram,

3111 Bristol Station Court, Carteret, New Jersey 07008, USA.

E-mail: sowmini.medavaram@gmail.com

\section{ACKNOWLEDGMENT}

Dr. Chirag Gandhi, neurosurgeon, and Dr. Leroy R. Sharer, pathologist, for their contributions.

\section{ONLINE SUPPLEMENT}

Supplementary data for this article are available online at jrheum.org.

\section{REFERENCES}

1. Stone JH, Chan JK, Deshpande V, Okazaki K, Umehara H, Zen Y IgG4-related disease. Int J Rheumatol 2013;2013:532612.

2. Lu LX, Della-Torre E, Stone JH, Clark SW. IgG4-related hypertrophic pachymeningitis: clinical features, diagnostic criteria, and treatment. JAMA Neurol 2014;71:785-93.

3. Hiraga A, Ozaki D, Tsuneyama A, Ito S, Koide K, Kuwabara S. Corticosteroid-responsive leptomeningitis with IgG4-positive plasma-cell infiltration. J Neurol Sci 2015;357:338-40.

4. Ferry JA, Klepeis V, Sohani AR, Harris NL, Preffer FI, Stone JH, et al. IgG4-related orbital disease and its mimics in a Western population. Am J Surg Pathol 2015;39:1688-700.

5. Wallace ZS, Khosroshahi A, Jakobiec FA, Deshpande V, Hatton MP, Ritter J, et al. IgG4-related systemic disease as a cause of "idiopathic" orbital inflammation, including orbital myositis, and trigeminal nerve involvement. Surv Ophthalmol 2012;57:26-33.

6. Mehta SH, Switzer JA, Biddinger P, Rojiani AM. IgG4-related leptomeningitis: a reversible cause of rapidly progressive cognitive decline. Neurology 2014;82:540-2.

7. Brito-Zeron P, Ramos-Casals M, Bosch X, Stone JH. The clinical spectrum of IgG4-related disease. Autoimmun Rev 2014; 13:1203-10.

8. Della-Torre E, Galli L, Franciotta D, Bozzolo EP, Briani C, Furlan $\mathrm{R}$, et al. Diagnostic value of IgG4 Indices in IgG4-related hypertrophic pachymeningitis. J Neuroimmunol 2014;266:82-6.

J Rheumatol 2016;43:4; doi:10.3899/jrheum.151236 